\title{
Medial canthal resection: an effective long-term cure for medial ectropion
}

\author{
T J Sullivan, J R O Collin
}

\begin{abstract}
The results of 37 medial canthal resection procedures performed for the correction of severe paralytic or involutional medial ectropion are presented with an average follow-up of 5.4 years. Epiphora was improved in 33 out of the 37 cases and all but one patient had an anatomically improved lid-globe apposition, medial canthal angle, and posterofixation of the medial canthus. These results confirmed the long-term value of the operation.
\end{abstract}

Adequate treatment of severe medial ectropion with medial canthal tendon laxity is difficult. Problems in surgery arise because the medial canthal tendon has a superficial and deep head, the posterior attachment of which is difficult to recreate. Further, the medial canthal tendon overlies the lacrimal canaliculus, and surgery to the medial canthal tendon may interfere with lacrimal drainage.

Various procedures ${ }^{1-3}$ have been described for medial ectropion. However, when there is a laxity of the medial canthal tendon, they will not improve epiphora, because the medial canthal tendon is not shortened sufficiently or posterofixated adequately.

$\mathrm{McCord}^{4}$ described a canaliculostomy procedure when treating neoplastic disease of the medial lower lid. Crawford et $a l^{5}$ then described a procedure for the correction of paralytic ectropion. This procedure involves resection of the medial canthal structures with a horizontal lid shortening, reconstituting the posterior limb of the medial canthal tendon with a permanent suture, and marsupialisation of the cut inferior canaliculus into the conjunctival sac of the lower fornix.

Since the original description for paralytic ectropion 37 procedures on 29 patients have been performed, not only for paralytic ectropion but also for involutional medial ectropion associated with medial canthal tendon laxity.

This paper reviews our long-term results from 37 medial canthal tendon resections. Factors to be considered for an acceptable result include relief from epiphora, elimination of exposure, and satisfactory cosmesis.

\section{Subjects and methods}

The records of 29 patients who had undergone medial canthal resection surgery at Moorfields Eye Hospital were reviewed retrospectively. There was a total of 37 procedures, which were bilateral in eight patients. In 17 patients the aetiology was paraly (Figs 1A, B) and in 12 involutional (Figs 2A, B). Sixteen were male and 13 female, with a mean age of 67.3 years at

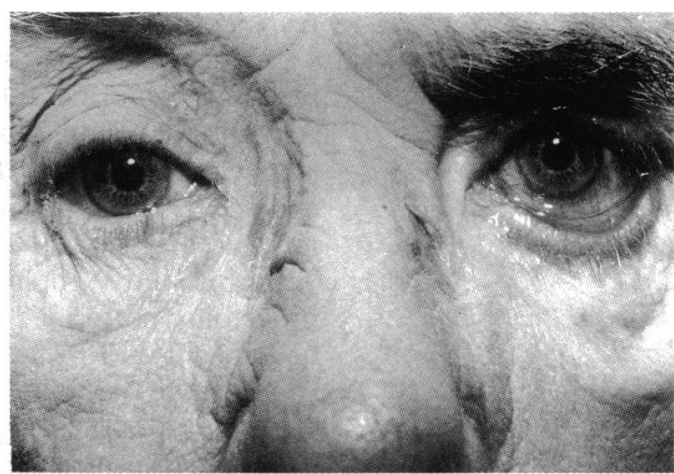

Figure IA Left paralytic ectropion (note brow droop).

operation. All the patients except one complained of epiphora. Seven patients also had symptoms of exposure; all of these had paralytic ectropions. Most patients had poor cosmesis, and this was more of a problem in the paralytic group.

In addition to a routine, full ocular examination the preoperative assessment included lid position, lid laxity, medial and lateral canthal tendon laxity, and patency and function of the lacrimal drainage apparatus. The indication for medial canthal tendon resection was a severe medial ectropion associated with marked laxity of the medial canthal tendon, where it was considered a simpler operation would fail or had already failed to achieve an acceptable result.

The operation was performed under either general or local anaesthesia. The lower lid margin was grasped immediately lateral to the punctum and pulled laterally. The lid was cut vertically through its full thickness (Fig 3a), including the medial canthal tendon and the inferior canaliculus, just lateral to the caruncle. Although the canaliculus was cut in this step, it was important to retain as much of it as possible for its later marsupialisation by placing a probe in the canaliculus and moving the scissors laterally after the initial vertical incision had been made through the skin and conjunctiva. The conjunctival aspect of this incision was contined retroplically (Fig

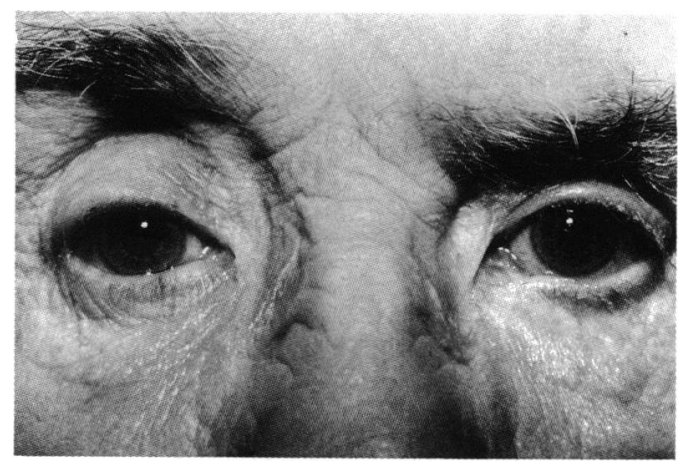

Figure IB Left paralytic ectropion 18 months after medial canthal resection. 


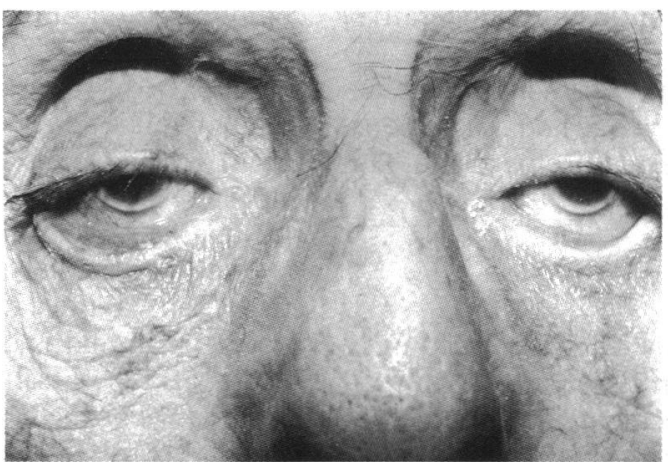

Figure 2A Bilateral involutional ectropion.

3b). Blunt ended scissors were then used to enter this retroplical plane. The tissues were spread with the blades tangential to the globe and directed to the posterior lacrimal crest. This avascular space was enlarged, and the medial orbital wall was exposed with two small malleable retractors.

Both half circle needles of a double armed 5-0 non-absorbable suture were placed through the periosteum of the posterior lacrimal crest, one at the level of the medial canthal tendon and one $2 \mathrm{~mm}$ higher (Fig $3 \mathrm{~b}, \mathrm{c}$ ). The lateral edge of the cut lid margin was then drawn towards the posterior lacrimal crest to assess the amount of horizontal lid shortening required. The lid was then shortened, and the two ends of the nonabsorbable suture were placed through the upper and lower aspects of the cut tarsal plate. These were not tied at this stage. The canalicular stump was cut to give small anterior and posterior flaps, which were used to fashion a new ostium. The anterior flap was sutured high up on the cut edge of the tarsus with two 8-0 absorbable sutures (Fig 3d). The lid margin was held medially as the permanent suture was tied to approximate the lower lid against the globe and recreate the

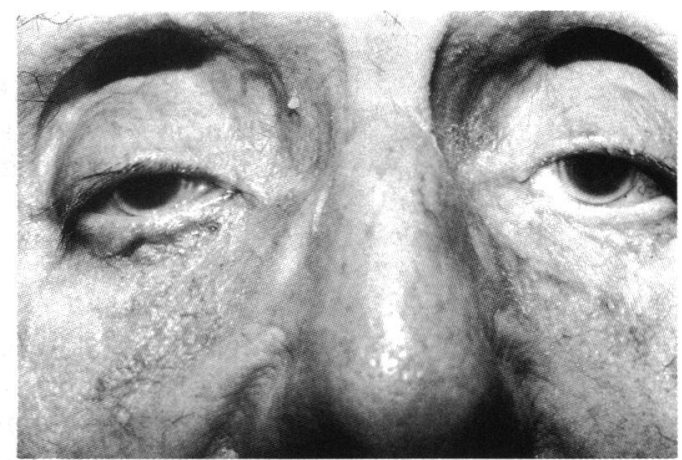

Figure 2B Bilateral involutional ectropion 12 months after medial canthal resections.

medial canthal angle. The lid margin and skin were then sutured with 6-0 silk (Fig $3 \mathrm{~d}$ ). It was occasionally necessary to resect the lower half of the caruncle if it prolapsed forward excessively. The eye was padded for 48 hours to minimise postoperative swelling. Skin sutures were removed five days postoperatively, and the lid margin suture was left for 10 days.

When the operation was originally described, the inferior canalicular remnant and the superior canaliculus were intubated with silicone tubing and the tube was left in situ for three to six months. We found that this was not necessary to achieve a patent lacrimal drainage system and no longer routinely intubate.

After the operation the patients were assessed subjectively and objectively for epiphora and exposure. Cosmesis was assessed subjectively, and by comparing pre- and postoperative appearances and anatomical lid-globe apposition. Initially all the patients underwent a postoperative Jones I test, syringing, and dacryocystogram to assess lacrimal drainage. As it became obvious that the procedure established a patent lacrimal drainage apparatus, formal syringing and the dacryocystogram have been
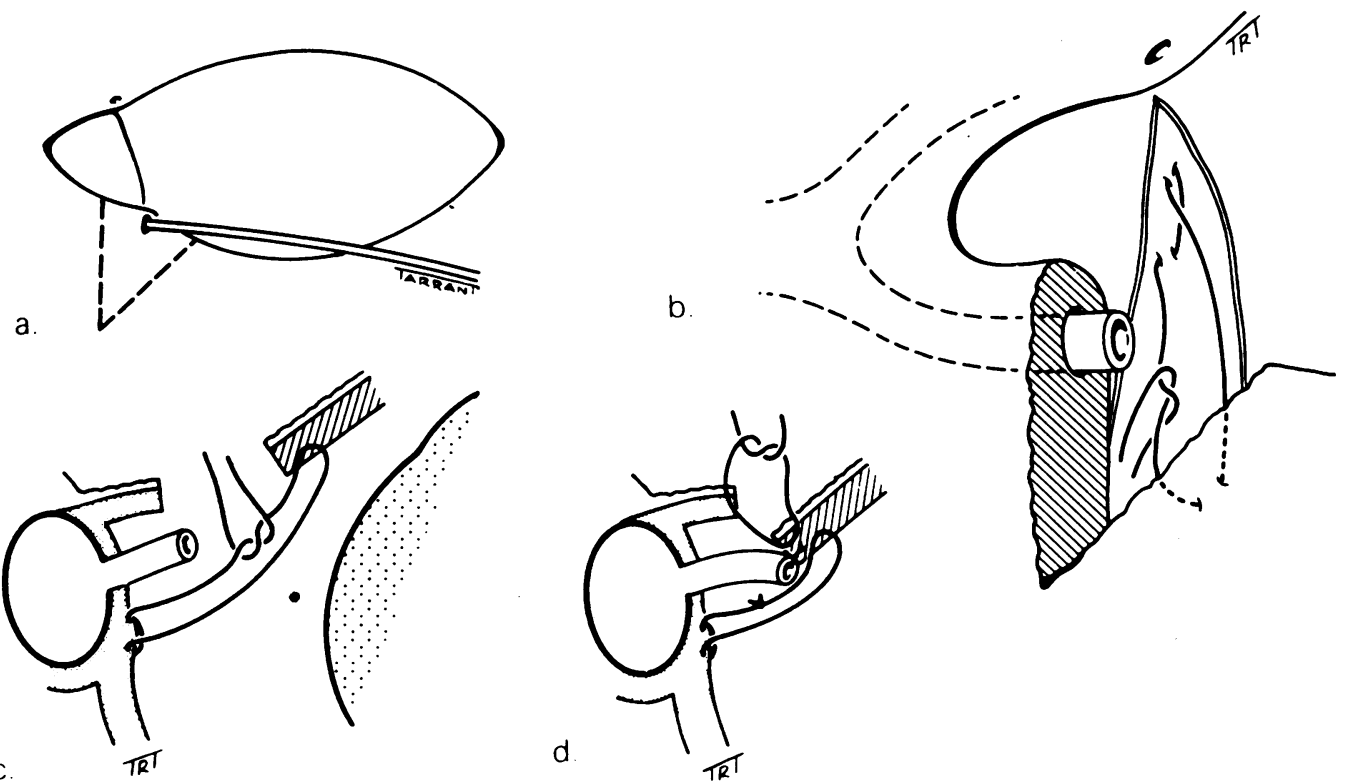

Figure 3 Diagrams of medial canthal resection surgery (taken with permission from $\mathcal{F} R O$ Collin: A manual of systematic eyelid surgery. 2nd ed. London: Churchill Livingstone, 1989).

$a$ Anterior view of full thickness lid to be resected. $b$ Anterior view after vertical incisions through lid showing conjunctiva opened behind the plica and posterior fixation suture through periosteum of posterior lacrimal crest. c Superior view of cross section diagram showing posterior fixation suture attached to medial end of resected eyelid.d Superior view of cross sectional diagram showing inferior canaliculus marsupialised into conjunctival sac and attached to superior edge of resected lid with skin closure suture in place. 
omitted from the routine postoperative examination.

Follow-up ranged from two months to nine years, mean $5 \cdot 4$ years, allowing valid conclusions to be drawn on the long-term effectiveness of the procedure.

\section{Results}

Epiphora was improved by 33 of 37 procedures. It was totally eliminated in 19 of the 37 . Of those patients with an improvement in epiphora all said the residual epiphora was mild and acceptable. Of the three patients (four procedures) in whom epiphora was not improved two had preexisting blockage of the common canaliculus. We have noted this to occur in paralytic ectropion, presumably from disuse secondary to loss of the lacrimal pump function. One of these patients has undergone conjunctivo dacryocystorhinostomy bypass surgery, and the epiphora has been eliminated. The other patient is awaiting lacrimal bypass surgery. In two of these patients the aetiology was paralytic and in the other involutional.

Seven of the 27 patients had exposure problems on presentation. In all of these the aetiology was paralytic. The exposure was eliminated in two, and four of the seven had marked improvement in symptoms and signs with intermittent lubricants. The one patient who did not improve later underwent a lateral tarsorrhaphy with good relief of symptoms.

Twenty one of the 29 patients were recorded in the notes as having felt the cosmetic appearance to be improved by the operation. One patient in whom the aetiology was paralytic felt that her cosmesis was not improved.

Anatomically the lid-globe apposition, medial canthal angle, and postero-fixation of the medial canthus were improved in 28 of 29 patients.

\section{Discussion}

The results of this study show that medial canthal tendon resection is an effective, long lasting cure for the problem of severe medial ectropion with medial canthal tendon laxity. Patients with medial ectropion present with epiphora, and the aim of surgery should be to correct the anatomical defect and to relieve the patient's symptoms. In our study epiphora was improved by 33 of 37 procedures performed. No other procedure shortens the tendon effectively, postero-fixates the medial canthus with good lid to globe apposition, and re-establishes a patent lacrimal drainage system.

Attempts to shorten the anterior limb of the medial canthal tendon by plication ${ }^{36}$ invariably concertina an already non-functioning canaliculus. Poor lid-globe apposition occurs owing to the anteriorly directed pull on the lid. The Lee medial canthoplasty' is a good procedure for inverting the punctum. However, it will not improve functional tear drainage where there is medial canthal tendon laxity, because the canaliculus is still long and lax. The lazy-T procedure ${ }^{2}$ is good for shortening the lid proper and inverting the punctum, especially if the lower lid retractors are caught in the horizontal tarso- conjunctival closure. When there is medial canthal tendon laxity, it will not alter tear drainage because the surgery is not directed to the anatomical defect.

Two recent papers have addressed the problem of postero-fixation in medial canthal malposition and marked medial ectropion. Jordan et $a l^{7}$ describe the medial tarsal strip procedure, an adaptation of the lateral tarsal strip. ${ }^{8}$ In this procedure the lid is cut vertically at the medial aspect of the tarsal plate and a tarsal strip fashioned. The medial canthal tissues, including canaliculus, are then excised, and the tarsal strip is sutured, ideally, to the frontal process of the maxilla (if the superior canaliculus is patent) or more posteriorly (if the patient does not have a patent canaliculus or lacrimal sac). This procedure effectively tightens the medial canthal tendon, and if the tarsal strip is sutured posteriorly enough will postero-fixate the medial canthus. In our experience, pull directed from the frontal process of the maxilla does not give as good lid-globe apposition as pull directed from the posterior lacrimal crest, which is the fixation point in our procedure. The medial tarsal strip procedure sacrifices the lower canaliculus, leaving the patient to rely on the superior canaliculus only for drainage. Our procedure confronts the problem of epiphora specifically by marsupialisation of the inferior canaliculus.

Edelstein and Dryden' reconstruct the medial canthal tendon in a complicated procedure, lifting a periosteal flap from the nasal bridge and passing it through a tunnel from the posterior lacrimal crest behind the common canaliculus parallel to the deep head of orbicularis to attach to medial tarsus. They consider possible lacrimal drainage dysfunction from kinking of the canaliculus or damage to the lacrimal sac. Their definition of an acceptable result does not include relief from epiphora. Essentially their procedure is another form of plicating the posterior limb of the medial canthal tendon, for which a good and easier technique has already been described. ${ }^{10}$ We use this technique when there is only mild medial canthal tendon laxity, and for this it works well. If there is more medial canthal tendon laxity, then medial canthal resection gives much better results.

Medial canthal resection has three aims, and aspects of the operation are directed to correcting the pathology in each case. Firstly, the medial canthal tendon is shortened directly. Secondly, it is postero-fixated to the posterior lacrimal crest. Thirdly, the cut inferior canaliculus is marsupialised to maintain lacrimal patency.

Following McCord's ${ }^{4}$ description of canaliculostomy, the procedure was first presented by Crawford et $a l^{5}$ in 1984. It has been used now for over nine years in 37 procedures. Our results presented here show that the procedure deals effectively with the problems of medial ectropion with medial canthal tendon laxity in most cases. The follow-up (mean 5.4 years) shows that its cure is effective in the long term in both paralytic and involutional medial ectropion.

We recommend medial canthal resection with marsupialisation of the inferior canaliculus as the procedure of choice for medial ectropion 
associated with moderate to marked medial canthal tendon laxity.

1 Lee OS. Operation for correction of everted lacrimal puncta. Am $\mathcal{F}$ Ophthalmol 1951; 34: 575-8.

2 Smith B. The 'lazy-T' correction of ectropion of the lower punctum. Arch Ophthalmol 1976; 94: 1149-50.

3 Stasior OG. Wendell L Hughes Lecture. Complications of ophthalmic plastic surgery and their prevention. Ophthalmology 1976; 81 OP: 543-52.

$4 \mathrm{McCord} \mathrm{CD}$. Canalicular resection and reconstruction by canaliculostomy. Ophthalmic Surg 1980; 11: 440-5.
5 Crawford GJ, Collin JRO, Moriarty PAJ. The correction of paralytic medial ectropion. Br f Ophthalmol 1984; 68: 63941.

6 Collin JRO. A manual of systematic eyelid surgery. 2nd ed. London: Churchill Livingstone, 1989: 32.

7 Jordan DR, Anderson RL, Thiese SM. The medial tarsal strip. Arch Ophthalmol 1990; 108: 120-4.

8 Anderson RL, Gordy DD. The tarsal strip procedure. Arch Ophthalmol 1979; 97: 2192-6.

9 Edelstein JP, Dryden RM. Medial palpebral tendon repair for medial ectropion of the lower eyelid. Ophthalmic Plast Reconstr Surg 1990; 6: 28-37.

10 Collin JRO. A manual of systematic eyelid surgery. 2nd ed. London: Churchill Livingstone, 1989; 33. 